\title{
Realización de un termostato inteligente utilizando la plataforma Raspberry PI como proyecto integrador de la materia de programación avanzada
}

\section{Realization of an intelligent thermostat using the Raspberry PI platform as an integrated project for the subject of advanced programming}

CHÁVEZ-VEGA, Nancy Beatriz†*, MARTÍNEZ-GARCÍA, Martha Lorena, ARZABALACONTRERAS, Ernesto Alonso y ESPINOZA-LUNA, Jorge Alberto

Universidad Tecnológica de Chihuahua, Av. Montes Americanos, No. 9501, Sector 35, C.P. 31216

ID $1^{\text {er }}$ Autor: Nancy Beatriz, Chávez-Vega / ORC ID: 0000-0001-8868-4387, Researcher ID Thomson: S-6751-2018, CVU CONACYT ID: 349472

ID $1^{\mathrm{er}}$ Coautor: Martha Lorena, Martínez-García / ORC ID: 0000-0003-0506-1809, Researcher ID Thomson: S-67622018, CVU CONACYT ID: 522690

ID $2^{\text {do }}$ Coautor: Ernesto Alonso, Arzabala-Contreras / ORC ID: 0000-0002-5121-0417, Researcher ID Thomson: S-67492018, CVU CONACYT ID: 254056

ID $3^{\text {er }}$ Coautor: Jorge Alberto, Espinoza-Luna / ORC ID: 0000-0002-1413-585X, Researcher ID Thomson: S-6825-2018, CVU CONACYT ID: 522655

DOI: $10.35429 / J O C T .2019 .10 .3 .7 .11$

Recibido 22 de Marzo, 2019; Aceptado 30 Junio, 2019

\section{Resumen}

El Aprendizaje Orientado a Proyectos es una metodología de enseñanza, donde los estudiantes generan su propio aprendizaje, desarrollando un producto que permita aplicar los saberes adquiridos, poniendo en práctica su conocimiento para resolver problemáticas reales del entorno. La herramienta educativa permite que el alumno desarrolle habilidades como el trabajo interdisciplinario y resolución de problemas y a la vez que dé respuesta a la demanda científica y social actual creando una transformación innovadora en el proceso educativo. Este artículo presenta el proceso de desarrollo de un termostato inteligente utilizando la plataforma Raspberry PI, y tecnologías del internet de las cosas IoT, orientado a la formación de estudiantes de nivel Técnico Superior Universitario, inscritos en noveno cuatrimestre de la carrera de Mecatrónica. La creación de este proyecto permite el cumplimiento de la competencia especifica de la asignatura, "Desarrollar proyectos de automatización y control, a través del diseño, la administración y la aplicación de nuevas tecnologías para satisfacer las necesidades del sector productivo".

Aprendizaje basado en proyectos, Competencias educativas, Interdisciplinario

\begin{abstract}
Project Oriented Learning (POL) is a teaching methodology, where students have their own learning, they devoped a product that allows them to apply and show the acquired knowledge. POL as an educational tool allows the student to develop skills such as interdisciplinary work and problem solving to tackle current scientific and social demands while creating an innovative transformation in the educational process. This article presents the process of developing an intelligent thermostat using the Raspberry PI platform, and Internet of Things IoT technologies, aimed at training students at the level of a Technical College, enrolled in the ninth quarter of the Mechatronics degree curriculum. The creation of this project allows the fulfillment of the specific competence of the degree, which is "Develop automation and control projects, through the design, administration and application of new technologies to meet the needs of the productive sector".
\end{abstract}

Project-based learning, Educational skills, Interdisciplinary

Citación: CHÁVEZ-VEGA, Nancy Beatriz, MARTÍNEZ-GARCÍA, Martha Lorena, ARZABALA-CONTRERAS, Ernesto Alonso y ESPINOZA-LUNA, Jorge Alberto. Realización de un termostato inteligente utilizando la plataforma Raspberry PI como proyecto integrador de la materia de programación avanzada. Revista de Tecnologías Computacionales. 2019. 3-10: 7-11

$\dagger$ Investigador contribuyendo como primer autor. 


\section{Introducción}

La retícula de la carrera de Ingeniero en Mecatrónica de las Universidades Tecnológicas en su noveno cuatrimestre tiene la opción de cursar una materia Optativa. En el caso de la Universidad Tecnológica de Chihuahua la materia Optativa seleccionada es la de Programación Avanzada.

Como el nombre de la materia lo indica se espera que al finalizar el curso el alumno aprenda metodologías y técnicas de programación más avanzadas y diferentes a las aprendidas en las materias de lenguaje de programación y programación visual que previamente cursaron en la carrera de TSU. Mediante el desarrollo del presente trabajo se propone impactar positivamente incrementando el uso de la metodología de enseñanza de aprendizaje orientado a proyectos.

Una de las competencias que deben adquirir los alumnos es la de: "desarrollar proyectos de automatización y control, a través del diseño, la administración y la aplicación de nuevas tecnologías para satisfacer las necesidades del sector productivo". En estas materias de programación es común que se escoja alguna plataforma de desarrollo de microcontroladores para realizar la parte práctica del curso. Una de las plataformas más populares para implementar proyectos de automatización es la plataforma Raspberry PI, la cual es una microcomputadora con la capacidad de procesamiento y conectividad suficiente para realizar prototipos de dispositivos tipo IoT (Internet de las cosas).

El curso se lleva a cabo utilizando la técnica de aprendizaje orientado a proyectos (POL) buscando fortalecer los conocimientos previos de programación y diseño electrónico y mecánico con el que cuentan los alumnos y que adquieran nuevos conocimientos de una manera guiada pero independiente por medio de la realización de un proyecto integrador de la clase. Dicho proyecto integrador se plantea en conjunto con la academia de docentes buscando que contengan los elementos de diseño electrónico, mecánico y de programación requeridos por los alumnos para demostrar que han alcanzado la competencia mencionada anteriormente.
El proyecto integrador a realizar es un Termostato Inteligente con conexión a Internet. La razón de escoger este producto como proyecto es que además de cumplir con los requisitos revisados por la academia de profesores representa un gran interés para los alumnos debido a su curiosidad acerca de los temas de dispositivos para el internet de las cosas, ya que un termostato inteligente es un producto con el cual se pueden relacionar de forma cotidiana tanto como usuario y como desarrolladores. La descripción de esta investigación consta de cuatro secciones: La primera contempla los objetivos, hipótesis, análisis de la problemática actual; la segunda define el marco teórico que fundamenta la investigación; en la tercera se observa la metodología utilizada y en la última sección los resultados y conclusiones obtenidos.

\section{Análisis del Problema}

El aprendizaje basado en proyectos (POL) se fundamenta en una filosofía que establece (Martin Pérez, 2002) que los conceptos deben de ser comprendidos a través de la observación y el contacto directo con las cosas, por lo que esta técnica tiene como elemento esencial conducir a los alumnos a la construcción de su conocimiento a partir del desarrollo de algún producto tangible aplicado a una problemática real. La aplicación de esta metodología de aprendizaje durante el desarrollo de la materia Optativa tiene como problemática principal el encontrar un proyecto que despierte el interés del alumno y que permita cumplir con el resultado de aprendizaje marcado en el contenido del programa de la asignatura.

\section{Justificación}

El proceso de enseñanza actual debe reconocer el papel determinante del profesor (Montes de Oca Recío, 2011) en la identificación, la planificación y la instrumentación de estrategias docentes adecuadas para lograr la formación de los estudiantes. Hoy en día se tiene la necesidad de metodologías y técnicas de aprendizaje centradas en el alumno, lo cual exige enfocar la enseñanza como un proceso de orientación del aprendizaje, donde surjan las condiciones para que los estudiantes no solo se apropien de los conocimientos, sino que desarrollen habilidades, $\mathrm{y}$ adquieran estrategias que les permitan actuar de forma independiente, comprometida y creadora, para resolver los problemas a los que deberá enfrentarse en su futuro profesional.

CHÁVEZ-VEGA, Nancy Beatriz, MARTÍNEZ-GARCÍA, Martha Lorena, ARZABALA-CONTRERAS, Ernesto Alonso y ESPINOZA-LUNA, Jorge Alberto. Realización de un termostato inteligente utilizando la plataforma Raspberry PI como proyecto integrador de la materia de programación avanzada. Revista de Tecnologías Computacionales. 2019 
El uso de metodologías educativas innovadoras permite al estudiante aprender a tomar sus propias decisiones y actuar de forma independiente; mejora su motivación por aprender pues debido al hecho de desarrollar un proyecto tangible crea experiencias que enriquecen sus conocimientos, habilidades y actitudes favoreciendo a un aprendizaje integral que fomente un perfil investigador.

\section{Objetivo}

Implementar un termostato inteligente utilizando la plataforma Raspberry PI como proyecto integrador de la materia de programación avanzada para los alumnos de noveno cuatrimestre aplicando la metodología de enseñanza-aprendizaje basado en proyectos durante el periodo mayo-agosto 2019.

\section{Marco Teórico}

Un termostato es un dispositivo que podría definirse como la evolución natural del termómetro. En ambos casos, su función principal (Huber, 2019) es la de medir la temperatura de un determinado lugar. Sin embargo, el termostato añade otras importantes funcionalidades, empezando por ser capaces de controlar y regular dicha temperatura, aumentándola o disminuyéndola, dependiendo de si detecta que hace frío o calor en la vivienda. Esta definición, que puede parecer muy simple, en realidad es muy compleja en cuanto al funcionamiento del termostato. Por eso es conveniente conocer su funcionamiento, los tipos de termostatos que existen, y las características de cada uno para escoger el que mejor se adapte a cada situación.

Cabe destacar algunas de sus características básicas de funcionamiento, que todos los modelos comparten. Como es el hecho de que el termostato se encarga de abrir o cerrar el circuito eléctrico del sistema de calefacción o del de refrigeración según la temperatura que se quiera alcanzar. Además, cualquier termostato de temperatura realiza una serie de mediciones que van más allá de la temperatura interna para llegar a la mejor solución, como el nivel de humedad, la concentración de $\mathrm{CO} 2$, o la temperatura externa. Una técnica de control aplicada en los termostatos básicos es el llamado Todo o Nada (On/Off), el cual usa un algoritmo (Ogata, 2010) simple de activación y desactivación de algún actuador.
Solamente revisa si la variable de proceso está por encima o por debajo de un set point determinado. En términos prácticos, la variable manipulada o la señal de control del controlador cambia entre "totalmente ON" o totalmente OFF, sin estados intermedios. Este tipo de accionamiento provoca un control muy impreciso de la variable de proceso. En el caso particular de los termostatos, activa el aire frio si $(\mathrm{ON})$ la temperatura es mayor a la de referencia o set point y lo desactiva (OFF) cuando la temperatura es menor o igual al set point. Conforme la salida del controlador abre completamente o cierra completamente al actuador, la temperatura nunca se estabiliza en un valor dado que el actuador nunca está en un valor entre 0 y $100 \%$. En aplicaciones prácticas, se considera para el control ON/OFF un set point alto y bajo o una banda de control, dado que si solo se considera un set point el actuador oscilaría con demasiada frecuencia provocando desgaste prematuro.

Node-RED es una herramienta (NodeRED, 2019) de desarrollo basada en flujo para programación visual desarrollada originalmente por IBM para conectar dispositivos de hardware, API y servicios en línea como parte de Internet de las Cosas. Proporciona un editor de flujo basado en un navegador web, que puede usarse para crear funciones de JavaScript. Los elementos de las aplicaciones se pueden guardar o compartir para su reutilización. El tiempo de ejecución se basa en Node.js.

\section{Metodología de la Investigación}

\section{Investigación y Recopilación de la Información}

Antes de dar a conocer el proyecto a los alumnos se les aplica una encuesta con respuestas determinadas por la escala Likert, para detectar los conocimientos previos, así como sus intereses relacionados al desarrollo de sistemas embebidos. En la tabla 1 se muestran la encuesta utilizada.

La escala utilizada en la encuesta es la siguiente: Extremadamente interesado "EI", Muy interesado "MI", Algo interesado "AI", No tan interesado "NI", Poco interesado "PI". 


\begin{tabular}{|c|c|c|}
\hline Pregunta & $\begin{array}{lll}\text { E } & \text { M } & \text { A } \\
\text { I } & \text { I } & \text { I }\end{array}$ & \\
\hline 1. Estas interesado en los sistemas embebidos & & \\
\hline $\begin{array}{l}\text { 2. Qué interés tienes por el IoT (Internet de } \\
\text { las cosas) }\end{array}$ & & \\
\hline $\begin{array}{l}\text { 3. Que tan interesado estas en usar un } \\
\text { hardware diferente a los microcontroladores } \\
\text { PIC y Arduino }\end{array}$ & & \\
\hline $\begin{array}{l}\text { 4.Te gustaría conocer nuevas herramientas de } \\
\text { programación para desarrollar dispositivos } \\
\text { tipo IoT }\end{array}$ & & \\
\hline $\begin{array}{l}\text { 5. Te gustaría ser evaluado exclusivamente } \\
\text { por el desarrollo de un proyecto }\end{array}$ & & \\
\hline $\begin{array}{l}\text { 6. Te interesaría repasar los conceptos de la } \\
\text { materia de lenguaje de programación }\end{array}$ & & \\
\hline $\begin{array}{l}\text { 7. Te gustaría aprender como transmitir y } \\
\text { recibir datos a través de internet }\end{array}$ & & \\
\hline
\end{tabular}

Tabla 1 Preguntas de la encuesta

En la figura 1, se despliegan los resultados concentrados de la aplicación de la encuesta. De la gráfica se puede observar que el $52 \%$ del grupo tiene un gran interés en aprender nuevas tecnologías y ser evaluados por medio de un proyecto integrador. Solamente un $25 \%$ del grupo no se encuentra interesado y los alumnos restantes son indiferentes al tema.

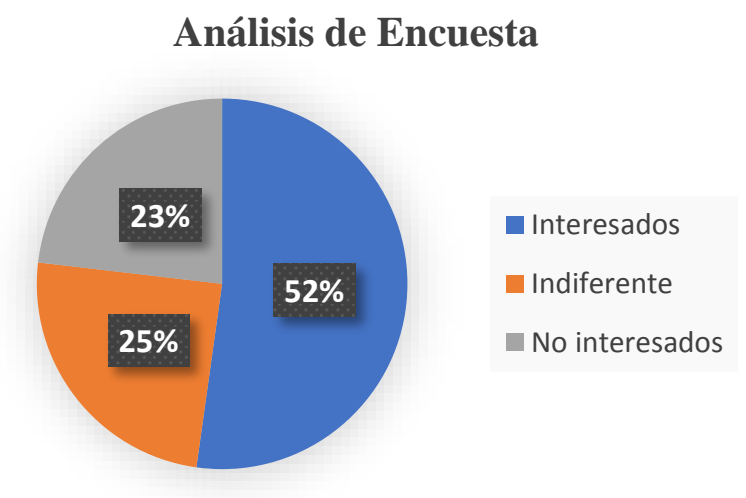

Figura 1 Análisis de Encuesta

\section{Diseño de la tablilla}

Para que el termóstato fabricado pueda ser compatible con las instalaciones eléctricas comunes ya existentes es necesario diseñar una tablilla que sirva como interfaz entre las conexiones del Raspberry PI y las conexiones eléctricas de una residencia para un termóstato comercial.

En la figura 2, se contempla un header de 40 pines macho hembra para la comunicación de todos los pines con la PCB elaborada.

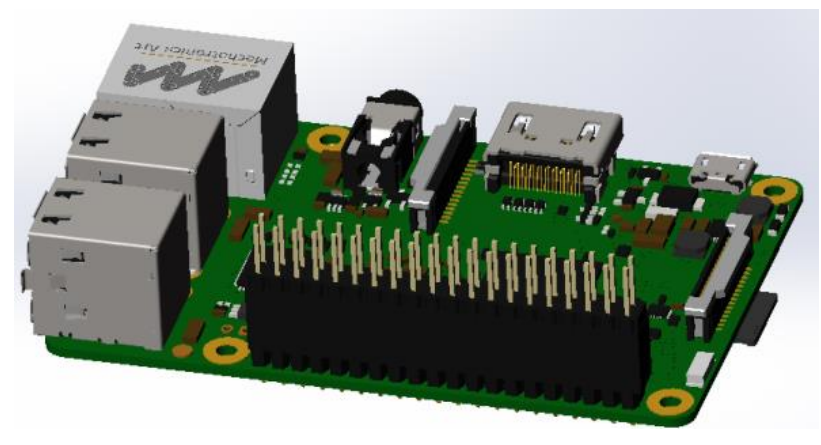

Figura 2 Raspberry en 3D

\section{Programación y Lógica de Control}

En este bloque se lee la señal proveniente de en este caso un Arduino, el cual manda los datos por comunicación serial, este lee el valor del sensor lo convierte en un número del 0-1023 y luego mediante código, este manda el valor de la temperatura hacia el Raspberry. El valor se guarda en una variable de tipo flow (variable de flujo de datos); que solo puede ser ingresada dentro del diagrama de código; para posteriormente utilizarla en algún otro bloque, además de desplegarla en un indicador visual dentro del panel principal. La variable de valor deseado es obtenida desde el panel principal (librería grafica de node red) la cual igualmente se almacena en una variable de tipo Flow (ver figura 3).



Figura 3 Bloque de código lectura de señal

Este pequeño lazo del programa (figura 4) sirve para detectar si el programa está en automático o simplemente se quiere mantener encendido el actuador. Dado que en el panel la lista de selección manda el dato solo una vez, se tiene que estar mandando constantemente para compararse siempre en el bloque siguiente; el bloque "msg" solo sirve para mantener un control del proceso y saber que se está haciendo. 


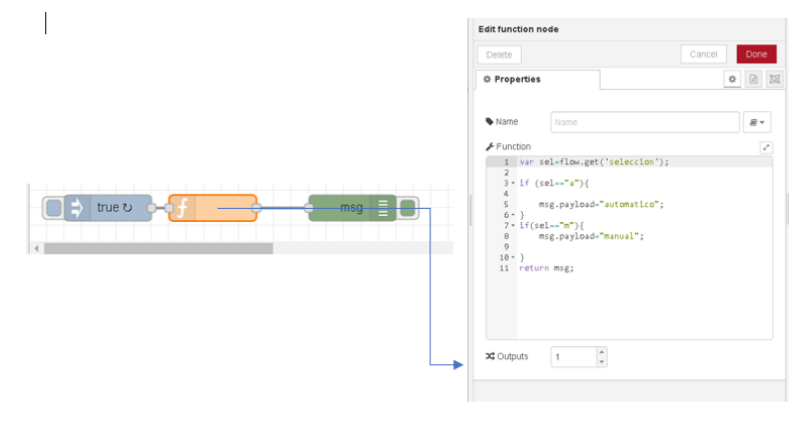

Figura 4 Bloque de código comparación

Para lograr almacenar la variable simplemente se utiliza este pequeño código mostrado en la figura 5. En este lazo se hacen las comparaciones de las variables para determinar si se necesita hacer alguna corrección, activación del actuador, entre otros.

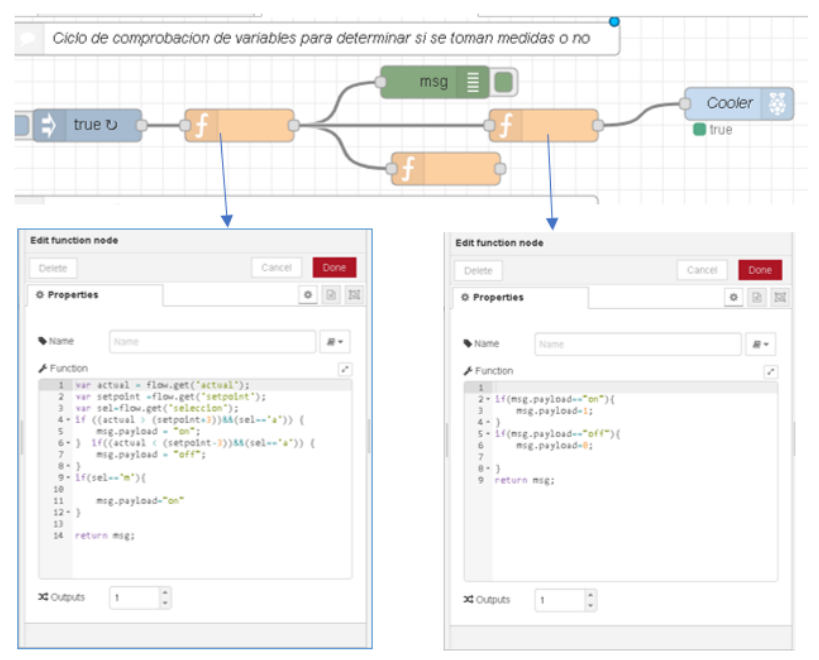

Figura 5 Bloque de código lazo principal

\section{Conclusiones y Resultados}

Como resultado en la figura 6 , se obtiene el ensamble del termostato y su aplicación de software para control de clima en el aula. Los estudiantes lograron conjuntar los conocimientos previos de diseño mecánico y diseño electrónico para la fabricación de un producto tangible resultado de las tareas realizadas al llevar a cabo el proyecto. Desde el punto de vista de la materia de programación lograron manejar diferentes lenguajes de programación y sus respectivas metodologías para desarrollar una aplicación que permite controlar un proceso e intercambiar datos a través del internet.



Figura 6 Imagen física del termostato

\section{Referencias}

Huber. (23 de 06 de 2019). Obtenido de Huberonline:

https://www.huberonline.com/download/manua 1s/BAL\%20CC\%20Einh\%C3\%A4nger\%20ES. pdf

Martin Pérez, M. (2002). El modelo educativo del Tecnológico de Monterrey. Monterrey: ISBN 968-891-055-4.

Montes de Oca Recío, E. M. (2011). Estrategias Docentes y Métodos de Enseñanza-Aprendizaje en la Educación Superior. Revista Humanidades Médicas.

Node-RED. (1 de Agosto de 2019).

Ogata, K. (2010). Ingeniería de Control Moderna. Madrid: Pearson Education. 